\title{
Improving Interferometry Instrumentation by Mixing Stereoscopy for $2 \pi$ Ambiguity Solving
}

Avi Karsenty, Department of Applied Physics/Electro-Optics Engineering, Faculty of Engineering, Lev Academic Center, Jerusalem, Israel

Yaron Lichtenstadt, Department of Applied Physics/Electro-Optics Engineering, Faculty of Engineering, Lev Academic Center, Jerusalem, Israel

Sagi Naeim, Department of Applied Physics/Electro-Optics Engineering, Faculty of Engineering, Lev Academic Center, Jerusalem, Israel

Yoel Arieli, Department of Applied Physics/Electro-Optics Engineering, Faculty of Engineering, Lev Academic Center, Jerusalem, Israel

\section{ABSTRACT}

Phase measurements obtained by high-coherence interferometry are restricted by the $2 \pi$ ambiguity to height differences smaller than $\lambda / 2$. A further restriction considers linear and nonlinear aberrations evolving in most interferometric systems due to the CCD-type array detectors. The authors present a new method to overcome the $2 \pi$ ambiguity in interferometry when using a stereoscopic approach. In this method, a reconstructed wavefront reflected from an object was propagated into two different angles to obtain two different images of the object. These two different images were subsequently processed by stereo algorithms to resolve the $2 \pi$ ambiguity. Such a method of wavefront propagation may enable several applications such as focusing and resolving the $2 \pi$ ambiguity, as described in the article.

\section{KEYWORDS}

$2 \pi$ Ambiguity, Coupling Techniques, Interferometric Measurements, Stereoscopy, Wavefront Propagation 


\section{INTRODUCTION}

\subsection{Coupling Techniques}

In the past decades, the world of measurements and instrumentation is rapidly growing, adapting itself to existing needs, and enabling the application domains, such as nanotechnology, space and remote sensing, chemistry and applied physics, metrology and more, to progress in parallel. Combining measurement techniques is a smart way to improve the specifications of such instrumentation. One domain, which is constantly progressing, is the interferometry technique when mixing it with other components and methods. The interferometer's architecture is usually dependent on the expected target to be measured, and on the final application. For example, improved accuracy and resolution could be obtained when mixing a Laser Diode (Norgia, 2007) to the interferometer system (Donati, 1996). On the other hand, amplitude fading phenomenon could be solved by self-mixing interferometry with piezo actuators, phase-sensing loop and liquid crystal attenuator (Norgia and Donati, 2003). More recently, displacement error phenomenon could be significantly decreased in displacement measuring interferometry by mixing FPGA Hardware-In-the-Loop (HIL) simulation (Wang, 2016). Another example is the coupling of laser Doppler sensors with interferometry enabling the study of the dynamic behaviour of a rotor (Dreier, 2013). Using existing proven techniques, and with the purpose of improving accuracy, we present a new approach to solve the $2 \pi$ ambiguity in interferometry by coupling stereoscopy.

\subsection{Interferometry's Achilles Heel}

The well-known $2 \pi$ ambiguity concern in interferometry has been addressed and solved in various ways over the last two decades. Many techniques have been developed to resolve or prevent the $2 \pi$ ambiguities in the interferometric measurements, such as multiple wavelength measurements (Lofdahl, 2001), phase unwrapping method (Yang, 2002), low-coherence interferometric differential phase measurements (Hitzenberger, 2001), phase-crossing technique (Yang, 2002), multiwavelength digital holography (Gass, 2003), terahertz phase imaging method with multiwavelengths (Zhang, 2006), and continuous-wave terahertz interferometry with multiwavelength phase unwrapping (Wang, 2010).

\subsection{The Motivation}

In this article, a new combined approach to overcome the $2 \pi$ ambiguity is presented, as shown in Figure 1. Highly accurate phase measurements are very important in applications of surface measurements, as presented in the last decades. Greivenkamp (1992) mentioned the need for Phase shifting interferometry in Optical Shop Testing (OST), Creath (1988) referred to the interferometry multiple techniques, and Arieli (2004) addressed the method for 3D measurement of MEMS structures. Nevertheless, phase measurements obtained by high coherence interferometry are restricted by the $2 \pi$ ambiguity to height differences smaller than $\lambda / 2$. In order to increase the dynamic 
Figure 1. Wavefront Propagation (WFP) diagram using stereoscopy

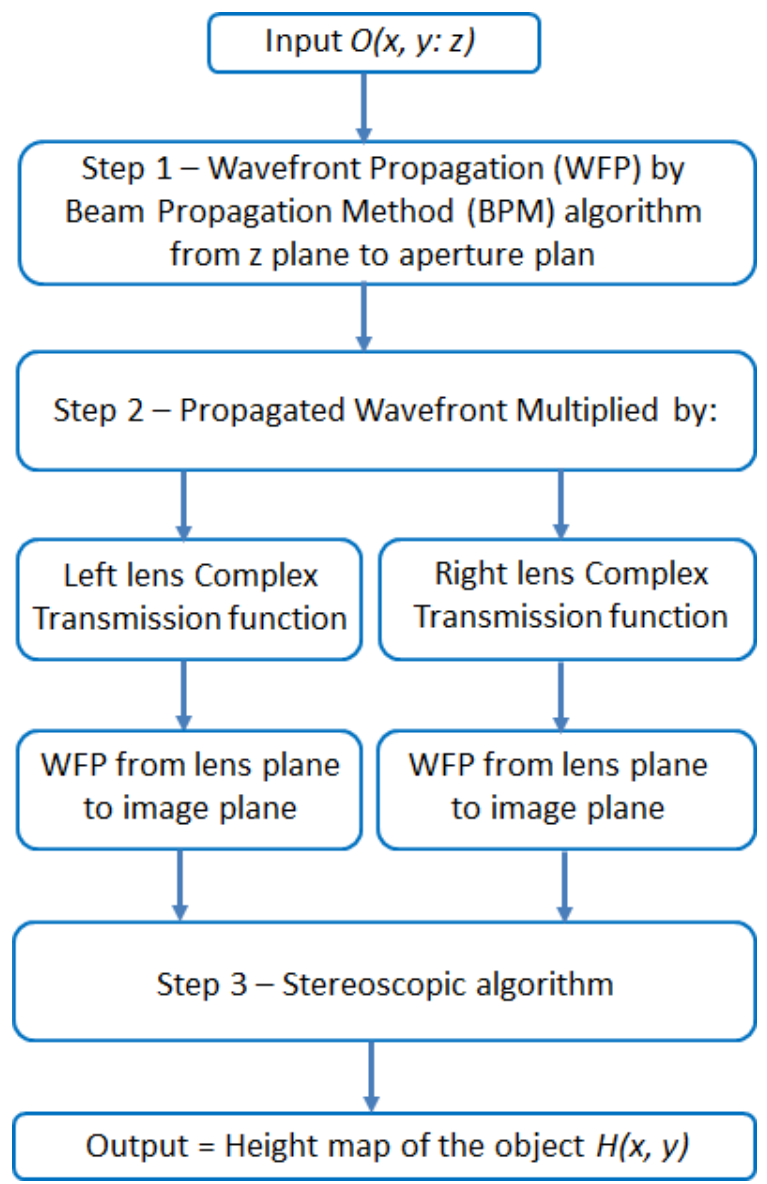

range of 3D measurements for continuous objects, known unwrapping algorithms can be used, as presented by Fornaro (1996) and Collaro (1998). However, for step-like objects, several different measurements, each consisting of a different wavelength, must be applied (Cheng, 1984). By comparing the phases obtained by these measurements at each point of the object, the $2 \pi$ ambiguity is resolved and a considerably larger dynamic range can be measured. In this article, we suggest to overcome this restriction by using a step-like object to digitally propagate the measured wavefront reflected by the object and refocusing it. By focusing each segment locally and by retaining the distances by which each part of the wavefront was propagated and focused, the information as to resolve the $2 \pi$ ambiguity is provided.

\section{METHODS AND RESULTS}

\subsection{Wavefront Propagation}

It is well-known that the reconstruction of a wavefront of an object directly from a hologram suffers from the $2 \pi$ ambiguity. However, the viewer can still see a clear, 
stereoscopic image of the object via the hologram. This fact suggests that the use of stereoscopic imaging may help to overcome the $2 \pi$ ambiguity. Thus, the stereoscopic information related to the depth of an object obtained from the reconstructed wavefront can be used to solve and overcome the $2 \pi$ ambiguity in a highly simple way. In this approach, one can create two separate virtual images of an object from its reconstructed wavefront, thereby presenting the object from two different angles, and one can subsequently use stereo algorithms to reconstruct its depth information without the $2 \pi$ ambiguity.

The creation of two virtual images of an object from its reconstructed wavefront requires a simulation of two imaging lens systems at different angles. This simulation may be achieved in three complementary steps:

- The first step is to propagate the reconstructed wavefront from the object plane to two identical imaging lenses of identical virtual imaging systems. This can be achieved using the Angular Spectrum Wave Front Propagation (ASWFP) as demonstrated by Arieli (2012) and Wolfling (2006);

- The second step is to multiply the propagated wavefront by a complex transmission function simulating two apertures shifted away from the optical axis, each associated with a virtual lens with a certain focal length (Stamnes, 1986);

- The third step is to propagate the modified wavefront to an image plane. This step may also be performed separately for each aperture. The best location of an image plane may be determined using the Gauss equation or by autofocus algorithms.

The two different images of an object obtained in the image plane may be processed by a stereoscopic algorithm to resolve the $2 \pi$ ambiguity.

\subsection{Step-by-Step Process}

To demonstrate the feasibility of this new method, we performed a complete simulation of the entire process. The aim of this simulation was to ensure that two different images of an object are obtained in the image plane, using this stereoscopic information to solve the $2 \pi$ ambiguity.

Figure 2 shows the two infinite long bars (top view) used for the simulation, which were laterally separated and located at different distances from the interference plane. The longitudinal distance between the two bars and the interference plane were chosen so that the phase shift between the two original wavefronts $\left(U_{1}\right.$ and $U_{2}$ ) from the bars would be an integer multiple of $2 \pi$ for the simulation wavelength. In such cases, the two bars could not be differentiated using fixed wavelength interferometry due to the $2 \pi$ ambiguity. Therefore, the simulation wavelength was set to $0.5 \mu \mathrm{m}$, and the plane $\mathrm{z}=0$ was chosen as the interference plane. The two bars were considered as the simulation object, in which one bar had a width of $50 \mu \mathrm{m}$ in the $\mathrm{X}$ dimension with its center located at the point $(-250,0,250)$ - respectively referring to $(\mathrm{x}, \mathrm{y}, \mathrm{z})$ - and the other bar had a width of $100 \mu \mathrm{m}$ in the $\mathrm{X}$ dimension with its center located at the point $(-500,0,500)$. Note that all dimensions were in microns. 
Figure 2. The simulated object

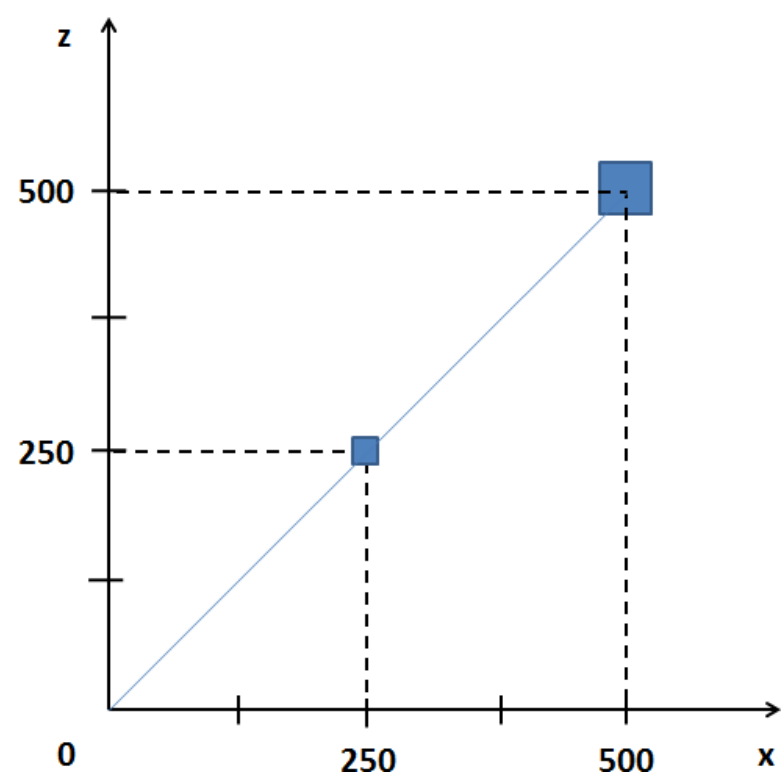

Thus, the original wavefront $O(x, y, z)$ from the object can be expressed as:

$$
o(x, y, z)=U_{1}\left(x, y: z_{1}\right)+U_{2}\left(x, y: z_{2}\right)=\operatorname{rect}\left(\frac{x}{\Delta_{1}}\right) * \delta\left(p_{1}\right)+\operatorname{rect}\left(\frac{x}{\Delta_{2}}\right) * \delta\left(p_{2}\right)
$$

where:

- $\mathrm{O}$ is the wavefront originated from the object that consists of two bars

- $\mathrm{U}_{1}$ is the wavefront originated from the bar located at $\mathrm{p}_{1}$

- $\mathrm{U}_{2}$ is the wavefront originated from the bar located at $\mathrm{p}_{2}$

- rect is the rectangular function (Yang, 2002)

- $\delta(\mathrm{p})$ is the Delta function located at the coordinates $\mathrm{p}=(\mathrm{x}, \mathrm{y}, \mathrm{z})$

- $\Delta_{1}=50.10^{-6}$

- $\Delta_{2}=100.10^{-6}$

- $\mathrm{p}_{1}=\left(250.10^{-6}, 0,-250.10^{-6}\right)$

- $\mathrm{p}_{2}=\left(500.10^{-6}, 0,-500.10^{-6}\right)$

The two parts $\mathrm{U}_{1}$ and $\mathrm{U}_{2}$ of the wavefront $\mathrm{O}$ were propagated separately to the interference plane at $\mathrm{z}=0 \mathrm{using}$ the ASWFP algorithm, i.e., the Fourier transform (Goodman, 2005) of each part of the wavefront:

$$
A_{1,2}\left(f_{x}, f_{y}: z_{1,2}\right)=A_{1,2}\left(\frac{\alpha}{\lambda}, \frac{\beta}{\lambda}\right)=\iint_{\infty} U_{1,2}\left(x, y: z_{1,2}\right) \exp \left[-i 2 \pi\left(\frac{\alpha}{\lambda} x+\frac{\beta}{\lambda} y\right)\right] d x d y
$$


Multiplying the two functions $A_{1,2}\left(f_{x}, f_{y}\right)$ by the phase factors would yield:

$$
A_{1,2}\left(f_{x}, f_{y}: 0\right)=A_{1,2}\left(\frac{\alpha}{\lambda}, \frac{\beta}{\lambda}: 0\right)=A_{1,2}\left(\frac{\alpha}{\lambda}, \frac{\beta}{\lambda}: 0\right) \exp \left[-i \frac{2 \pi}{\lambda} \sqrt{1-\alpha^{2}-\beta^{2}} z_{1,2}\right]
$$

where $\mathrm{z}_{1}=250 \mu \mathrm{m}$ and $\mathrm{z}_{2}=500 \mu \mathrm{m}$, and the inverse Fourier transformation of the functions $A_{1,2}\left(f_{x}, f_{y}: 0\right)$ to obtain the two propagated parts of the wavefront $U_{1,2}(x$, $y: 0)$ at the plane $\mathrm{z}=0$.

At the interference plane, the two propagated parts of the wavefronts $U_{1,2}(x, y: 0)$ were combined to present a reconstructed wavefront. In the second step, the combined wavefront was propagated through two virtual apertures, with each being associated with a virtual lens. The apertures were located in the interference plane and their sizes were chosen such that the two bars were visible from the right aperture, but the frontal bar covered the rear bar from the left aperture. This arrangement is presented in Figure 3. Results of the Fraunhofer diffraction through the aperture will be presented in Figure 4 and 5.

The complex transmission function that simulated the two shifted lens apertures can be described as:

$$
\tau(x, y)=\operatorname{circ}\left(\frac{r}{R}\right) \exp \left[\frac{i \pi}{\lambda f}\left(x^{2}+y^{2}\right)\right] *\left[\delta\left(p_{1}\right)+\delta\left(p_{2}\right)\right]
$$

where:

Figure 3. Positioning of the apertures (circles) vs. the eyes

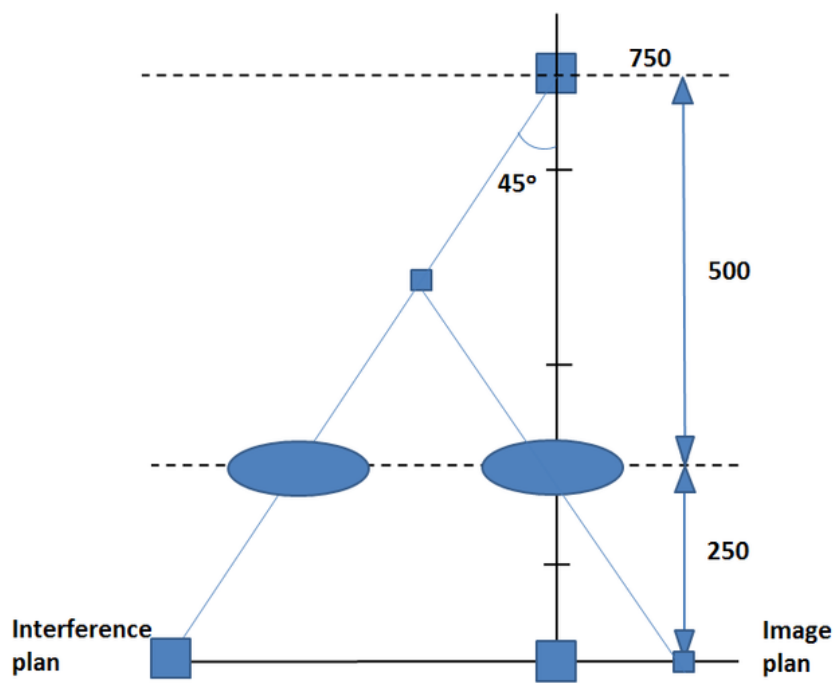


Figure 4. 3D simulation of the Fraunhofer diffraction through the aperture

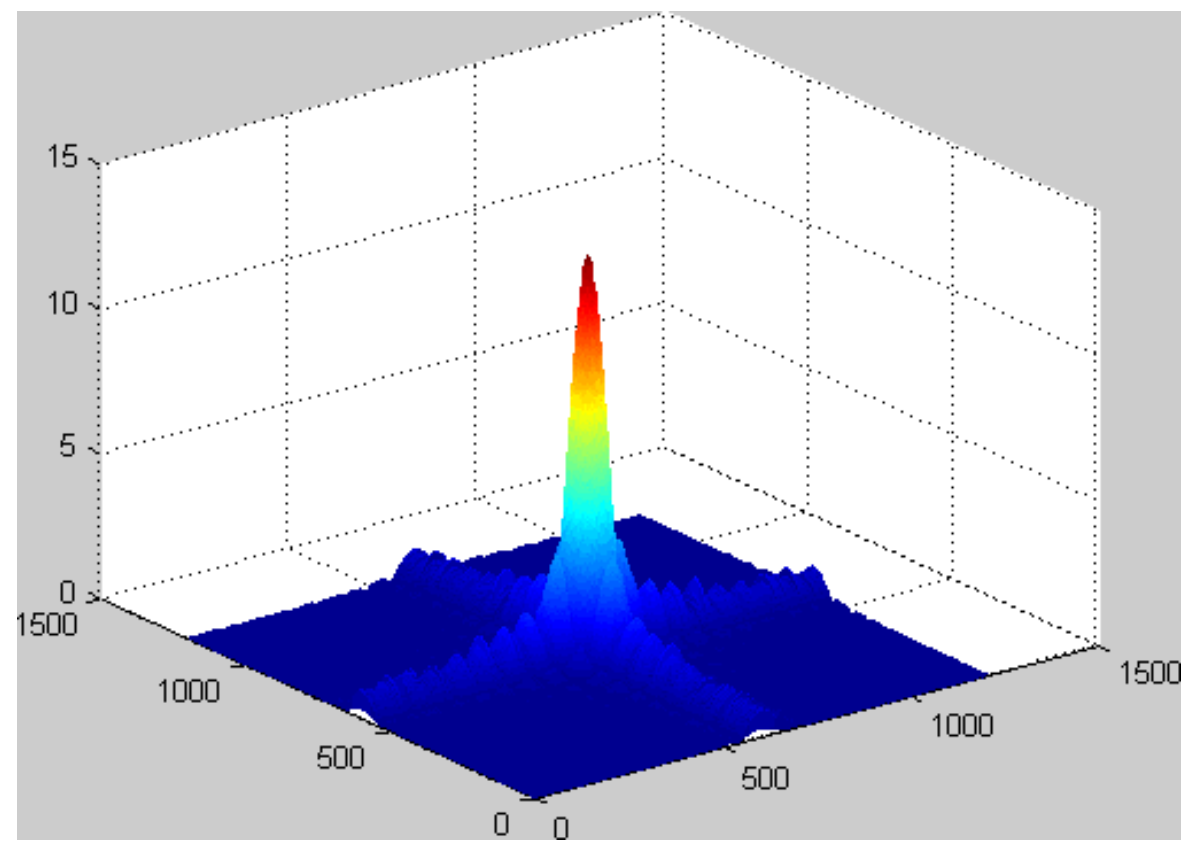

Figure 5. 2D simulation of the Fraunhofer diffraction through the aperture

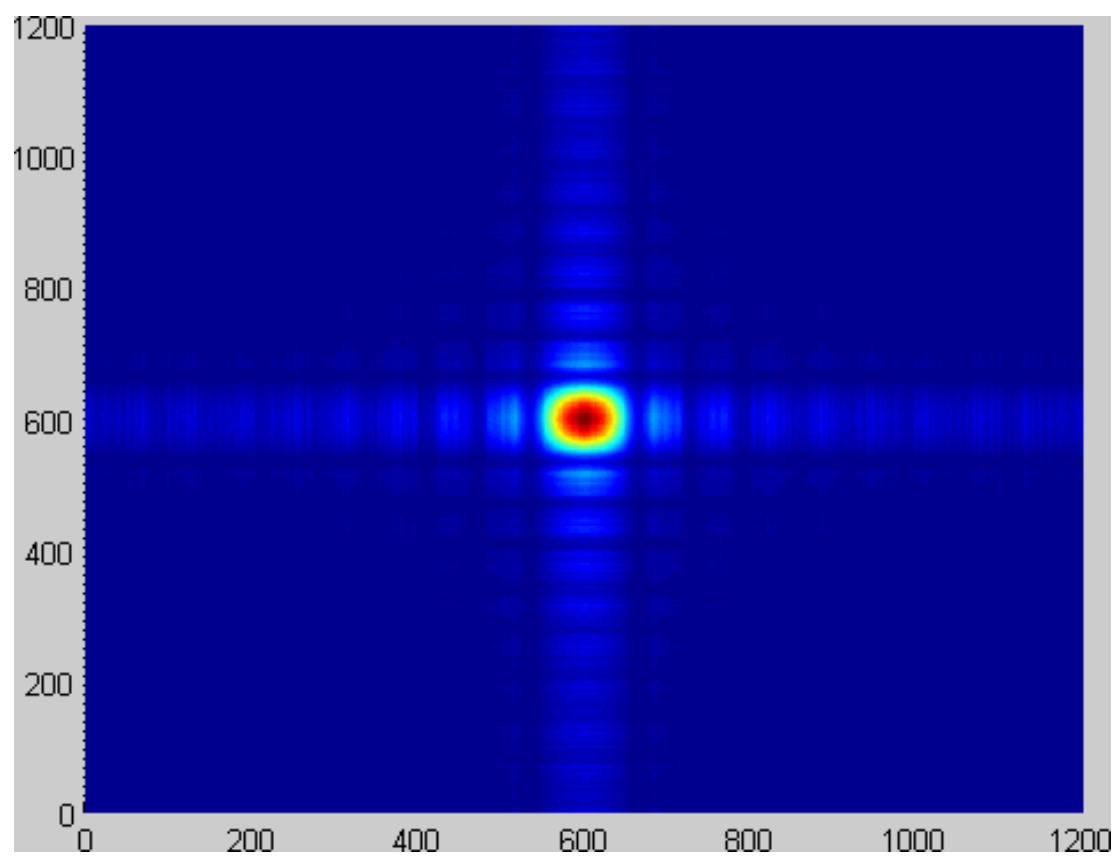


$R=250 \cdot 10^{-6}$

$f=125 \cdot 10^{-6}$

$p_{1}=(0,0,0)$

$p_{2}=\left(500 \cdot 10^{-6}, 0,0\right)$

After multiplying by the complex transmission function, the two parts of the wavefront were propagated again to the image plane. In this simulation, the wavefront was propagated separately from each aperture to the image plane, for which the location at $\mathrm{z}=250 \mu \mathrm{m}$ was determined using the Gauss equation:

$\frac{1}{f}=\frac{1}{z_{1}}+\frac{1}{z_{2}}$

where $\mathrm{z}_{1}=250 \mu \mathrm{m}$ and $\mathrm{f}=125 \mu \mathrm{m}$. The simulation results are shown in Figure 6 and Figure 7 , in which the image of the bars is rotated by $90^{\circ}$. Figure 6 shows the result of the wavefront propagation simulation via the right aperture, and Figure 7 shows the result of the wavefront propagation simulation via the left aperture.

Two rectangles were observed for the right aperture because both bars were visible as shown in Figure 6. However, for the left aperture, only one rectangle was observed (Figure 7) because the frontal bar covered the rear bar. In addition, it can be observed from Figure 7 that the image of the frontal bar had $1 \mathrm{X}$ magnification, whereas the rear bar appeared much wider in the image compared to its actual width. This effect

Figure 6. The result of the wavefront propagation simulation via the right aperture in which the frontal bar does not conceal the second

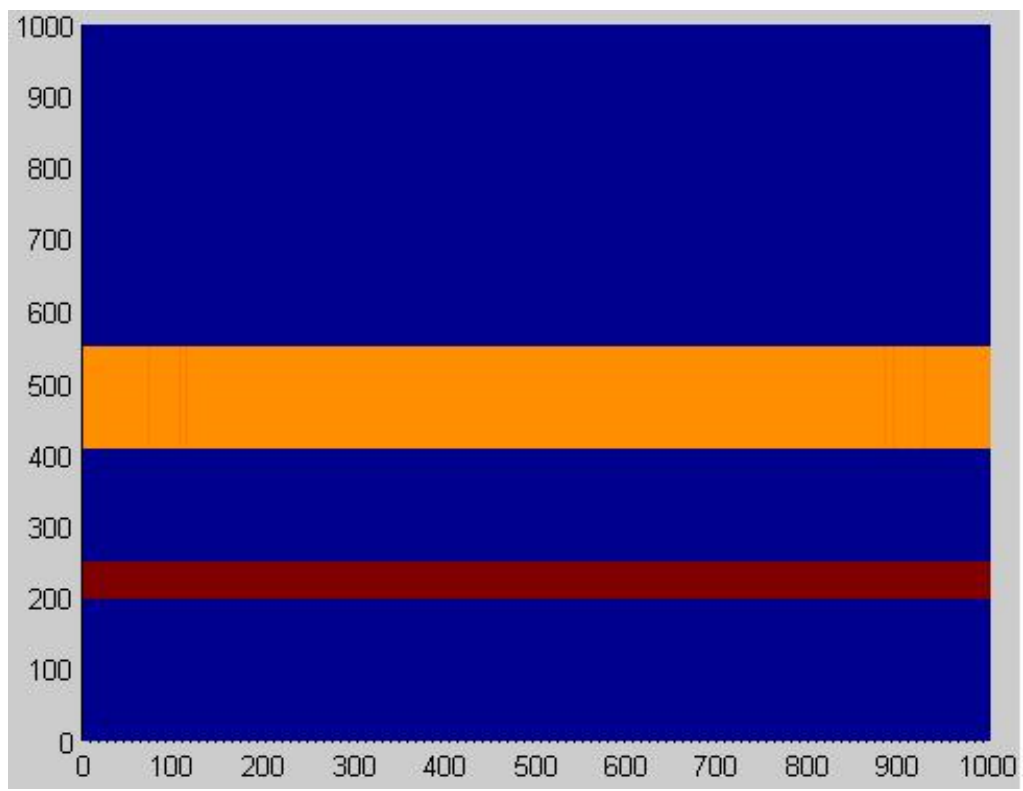


Figure 7. The result of the wavefront propagation simulation via the left aperture in which the frontal bar conceals the second bar

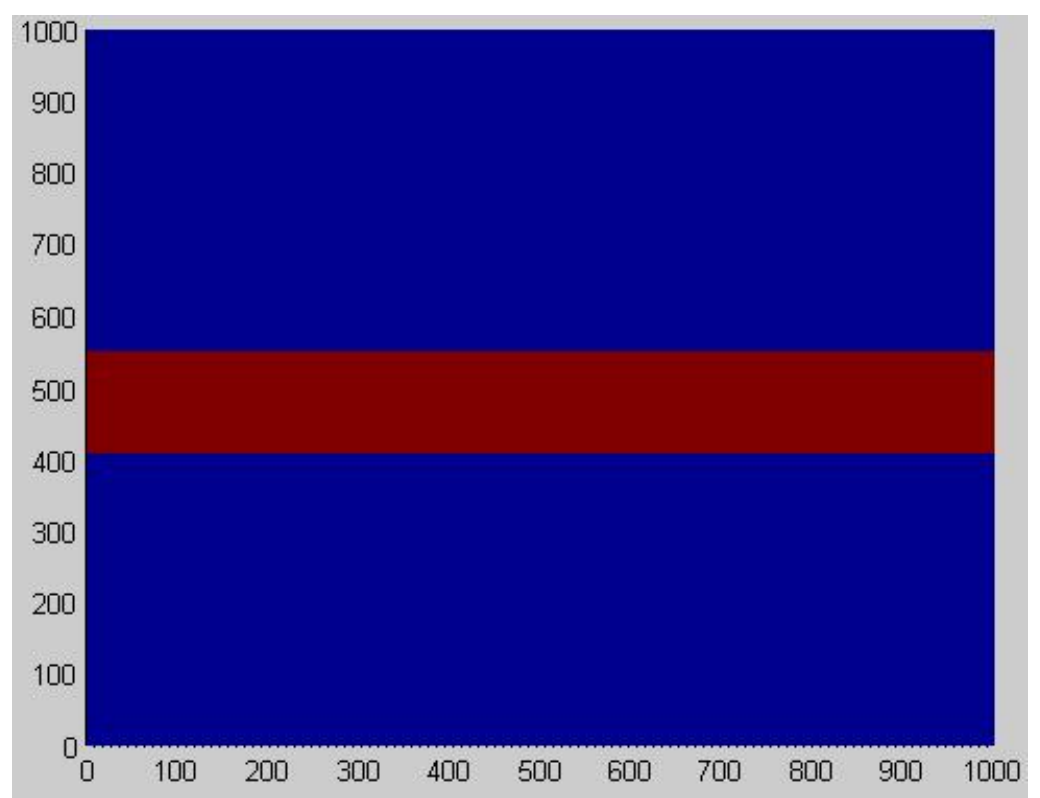

was observed because the best location of the image plane was calculated based on the frontal bar, while the rear bar had a different image plane that was considerably closer to the interference plane and was out of focus. These results show that by reconstructing the stereoscopic information about the depth of the object obtained from the reconstructed wavefront, the two bars can be differentiated despite the fact that the phase shift between the two original wavefronts from the bars is an integer multiple of $2 \pi$.

Thus, these results confirm that the reconstruction of the stereoscopic information about the depth of the object obtained from the reconstructed wavefront can overcome the $2 \pi$ ambiguity in spite of using fixed wavelength interferometry.

\section{DISCUSSION}

This new method is preferable over other existing solutions because it does not require any special equipment, such as multiwavelength lasers. In addition, the stereoscopic approach is more accurate when compared to other techniques using physical assumptions regarding the measurement period, such as unwrapping algorithms.

In order to emphasize the clear advantages of this new method, we present a table of the main published techniques solving the $2 \pi$ ambiguity. From Table 1 , it appears that most of the well-known techniques use complex experiments made of several hardware components. As a direct consequence of the required specific hardware, such methods are much more expensive and require accurate calibration of the setup, meaning more effort and time invested. 
Table 1. Selection of representative techniques solving the $2 \pi$ ambiguity

\begin{tabular}{|l|l|l|}
\hline \multicolumn{1}{|c|}{ Technique and Year } & \multicolumn{1}{|c|}{ Hardware and Software } & \multicolumn{1}{|c|}{$\begin{array}{c}\text { Advantages and } \\
\text { Disadvantages }\end{array}$} \\
\hline $\begin{array}{l}\text { Stereoscopy Mixing presented in this } \\
\text { article, (Karsenty, 2017) }\end{array}$ & $\begin{array}{l}\text { Any standard interferometer. Using Wavefront } \\
\text { Propagation Matlab program }\end{array}$ & $\begin{array}{l}\text { Low cost, no } \\
\text { additional HW, on-line } \\
\text { measurement }\end{array}$ \\
\hline $\begin{array}{l}\text { MPU (Multiwavelength Phase } \\
\text { Unwrapping), (Wang, 2010) }\end{array}$ & $\begin{array}{l}\text { 4 HDPE lenses L1, L2, L3, L4, 2 Beam Splitters } \\
\text { BS1, BS2, M1, M2, metallic mirrors; Stage1, } \\
\text { Stage2, Stage3, computer-controlled linear stages }\end{array}$ & $\begin{array}{l}\text { Requires specific and } \\
\text { complex HW }\end{array}$ \\
\hline $\begin{array}{l}\text { MWDH (Multi Wavelength Digital } \\
\text { Holography), (Gass, 2003) }\end{array}$ & $\begin{array}{l}\text { CCD Camera, HeNe laser 633 nm, doubled } \\
\text { Nd:YAG laser 532 nm, 3 BS (Beam Splitters) }\end{array}$ & $\begin{array}{l}\text { Requires specific and } \\
\text { complex HW }\end{array}$ \\
\hline $\begin{array}{l}\text { LCPC (Low coherence } \\
\text { Phase Crossing), (Yang, 2002) }\end{array}$ & $\begin{array}{l}\text { Modified Michelson interferometer, Ti Sapphire } \\
\text { laser 775nm, CW 1550nm light semiconductor } \\
\text { laser. }\end{array}$ & $\begin{array}{l}\text { Requires specific and } \\
\text { complex HW }\end{array}$ \\
\hline $\begin{array}{l}\text { CSRA (Candidate Solution Rejection } \\
\text { Algorithm), (Lofdahl 2001) }\end{array}$ & Complex algorithm code & $\begin{array}{l}\text { Requires post-measure } \\
\text { Data Processing }\end{array}$ \\
\hline
\end{tabular}

In comparison with existing complex solutions, the stereoscopic approach is considerably simpler and preferable because it does not require any special equipment, such as multiwavelength lasers. Moreover, this approach is more accurate than the other existing techniques that apply physical assumptions concerning the constraints of the measurement period, similar to temporal unwrapping methods.

\section{CONCLUSION}

We introduced a new method in order to solve the $2 \pi$ ambiguity and the nonlinearities due to detectors occurring in interferometric measurements. In this approach, two separated virtual images that introduce the object from two different angles using the reconstructed wavefront were obtained. Using stereoscopic algorithms for the two images obtained at different angles, the $2 \pi$ ambiguity could be overcome. To validate this approach, a simulation was performed in which two bars that cannot be differentiated in regular wavefront reconstruction were differentiated by reconstructing the stereoscopic information regarding the depth of the object obtained from the reconstructed wavefront.

We demonstrated and implemented the stereoscopic method, proving its promising usefulness. This article proves the efficiency of this technique in presence of $2 \pi$ ambiguities and other noise artifacts.

\section{ACKNOWLEDGMENT}

This research received no specific grant from any funding agency in the public, commercial, or not-for-profit sectors. 


\section{REFERENCES}

Arieli, Y., Wolfling, S., Lanzmann, E., Feigin, G., Kuzniz, T., \& Saban, Y. (2012). US Patent US 8,319,975 B2.

Cheng, Y. Y., \& Wyant, J. C. (1984). Two-wavelength phase shifting interferometry. Applied Optics, 23(24), 4539-4543. doi:10.1364/AO.23.004539 PMID:18213347

Collaro, A., Franceschetti, G., Palmieri, F., \& Ferreiro, M. S. (1998). Phase unwrapping by means of genetic algorithms. JOSA A, 15(2), 407-418. doi:10.1364/ JOSAA.15.000407

Creath, K. (1988). Phase measurements interferometry techniques. Progress in Optics, XXVI, 349-393. doi:10.1016/S0079-6638(08)70178-1

Donati, S., Falzoni, L., \& Merlo, S. (1996). A PC-Interfaced, Compact Laser-Diode Feedback Interferometer for Displacement Measurements. IEEE Transactions on Instrumentation and Measurement, 45(6), 942-947. doi:10.1109/19.543990

Dreier, F., Günther, P., Pfister, T., Czarske, J. W., \& Fischer, A. (2013). Interferometric Sensor System for Blade Vibration Measurements in Turbomachine Applications. IEEE Transactions on Instrumentation and Measurement, 62(8), 2297-2302. doi:10.1109/ TIM.2013.2255993

Fornaro, G., Franceschetti, G., Lanari, R., \& Sansosti, E. (1996). Robust phaseunwrapping techniques: A comparison. JOSA A, 13(12), 2355-2366. doi:10.1364/ JOSAA.13.002355

Gass, J., Dakoff, A., \& Kim, M. K. (2003). Phase imaging without $2 \pi$ ambiguity by multiwavelength digital holography. Optics Letters, 28(13), 1141-1143. doi:10.1364/ OL.28.001141 PMID:12879934

Goodman, J. W. (2005). Introduction to Fourier Optics. Stanford, USA: Roberts and Company Publishers.

Greivenkamp, J. E., \& Bruning, J. H. (1992). Phase shifting interferometry in Optical Shop Testing. New York, USA: Wiley.

Hitzenberger, C. K., Sticker, M., Leitgeb, R., Sattmann, H., \& Fercher, A. F. (2001). Overcoming the $2 \pi$ ambiguity in low-coherence interferometric differential phase measurements. In Proc. SPIE 4251, Coherence Domain Optical Methods in Biomedical Science and Clinical Applications (Vol. 81).

Lofdahl, M. G., \& Eriksson, H. (2001). An Algorithm for Resolving 2 $\pi$ Ambiguities in Interferometric Measurements by use of Multiple Wavelengths. Optical Engineering (Redondo Beach, Calif.), 40(6). 
Norgia, M., \& Donati, S. (2003). A Displacement-Measuring Instrument Utilizing Self-Mixing Interferometry. IEEE Transactions on Instrumentation and Measurement, 52(6), 1765-1770. doi:10.1109/TIM.2003.820451

Norgia, M., Giuliani, G., \& Donati, S. (2007). Absolute Distance Measurement With Improved Accuracy Using Laser Diode Self-Mixing Interferometry in a Closed Loop. IEEE Transactions on Instrumentation and Measurement, 56(5), 1894-1900. doi:10.1109/TIM.2007.904551

Stamnes, J. J. (1986). Waves in Focal Regions: Propagation, Diffraction and Focusing of Light, Sound and Water Waves. Bristol: A. Hilger.

Wang, C., \& Ellis, J. D. (2016). Data Age Error Compensation for Non-constant Velocity Metrology. IEEE Transactions on Instrumentation and Measurement, 65(11), 2601-2611. doi:10.1109/TIM.2016.2597998

Wang, X., Hou, L., \& Zhang, Y. (2010). Continuous-wave terahertz interferometry with multiwavelength phase unwrapping. Applied Optics, 49(27), 5095-5102. doi:10.1364/ AO.49.005095 PMID:20856283

Wolfling, S., Banitt, D., Ben-Yosef, N., \& Arieli, Y. (2004). Innovative metrology method for the 3-dimensional measurement of MEMS structures. In Proc. SPIE 5343, Reliability, Testing, and Characterization of MEMS/MOEMS III (pp. 255-263).

Wolfling, S., Lanzmann, E., \& Arieli, Y. (2006). Wavefront Analysis using Spatial Phase Shift imaging interferometry. Applied Optics, 45(12), 2586-2596. doi:10.1364/ AO.45.002586 PMID:16633406

Yang, C., Wax, A., Dasari, R. R., \& Feld, M. S. (2002). $2 \pi$ ambiguity-free optical distance measurement with subnanometer precision with a novel phase-crossing lowcoherence interferometer. Optics Letters, 27(2), 77-79. doi:10.1364/OL.27.000077 PMID: 18007717

Zhang, L., Zhang, Y., Zhang, C., Zhao, Y., \& Liu, X. (2006). Terahertz multiwavelength phase imaging without $2 \pi$ ambiguity. Optics Letters, 31(24), 3668-3670. doi:10.1364/ OL.31.003668 PMID:17130939 
Avi Karsenty received the PhD degree in Applied Physics /Material Science (Microelectronics and Electro-Optics) from the Hebrew University of Jerusalem in 2003. His research focuses on nanoscale electro-optics coupled-devices. After 22 years in High-Tech industries, part of which as Engineer and Manager for 16 years with Intel, he is today the Physics/Electro-Optics Engineering Department's Head. Avi Karsenty is an IEEE Senior Member and OSA Senior Member and received 38 Awards in Engineering/Physics.

Yaron Lichtenstadt received his BSc degree in Applied Physics / Electro-Optics Engineering Department from the Jerusalem College of Technology in 2013. MBA in Business Administration from Bar-llan university 2018.

Sagi Naeim received his BSc degree in Applied Physics / Electro-Optics Engineering Department from the Jerusalem College of Technology in 2013. Sagi is currently studying for MSc in Electrical Engineering at Tel-Aviv University.

Yoel Arieli received his PhD Degree in Applied Physics \& Material Science (Electro-Optics Division) from the Hebrew University of Jerusalem (HUJI), Israel. He is the former Head of the Applied Physics/Electro-Optics Engineering Department at the Lev Academic Center. His expertise is in Spectroscopy, Interferometry, and Imaging, and his interests of research are OCT, Hyperspectral imaging, and Thermography. He has professional experience in CTO for 15 years and as a Consultant for 10 years. 